\title{
Erratum to: Diet of young Atlantic bluefin tuna (Thunnus thynnus) in eastern and western Atlantic foraging grounds
}

John M. Logan • Enrique Rodríguez-Marín • Nicolas Goñi •

Santiago Barreiro · Haritz Arrizabalaga $\cdot$ Walter Golet · Molly Lutcavage

Published online: 6 August 2011

(C) Springer-Verlag 2011

Erratum to: Mar Biol (2011) 158:73-85

DOI 10.1007/s00227-010-1543-0

The $y$-axis and legend of Fig. 4 were published with incorrectly labeled error bar units in the original version of the article. The corrected figure and caption are produced in the following page.

The online version of the original article can be found under doi:10.1007/s00227-010-1543-0.

J. M. Logan · W. Golet · M. Lutcavage

Large Pelagics Research Center,

Department of Biological Sciences,

University of New Hampshire, Durham, NH 03824, USA

J. M. Logan ( ()

Massachusetts Division of Marine Fisheries,

1213 Purchase Street, New Bedford, MA 02740, USA

e-mail: john.logan@state.ma.us

E. Rodríguez-Marín · S. Barreiro Instituto Español de Oceanografía (IEO), Centro Oceanográfico de Santander, Apartado 240, 39080 Santander, Spain

N. Goñi · H. Arrizabalaga

AZTI Tecnalia, Herrera Kaia Portualdea z/g,

20110 Pasaia, Gipuzkoa, Spain

Present Address:

M. Lutcavage

Large Pelagics Research Center, Department of Natural

Resources Conservation, University of Massachusetts Amherst,

108 East. Main Street, Gloucester, MA 01930, USA 

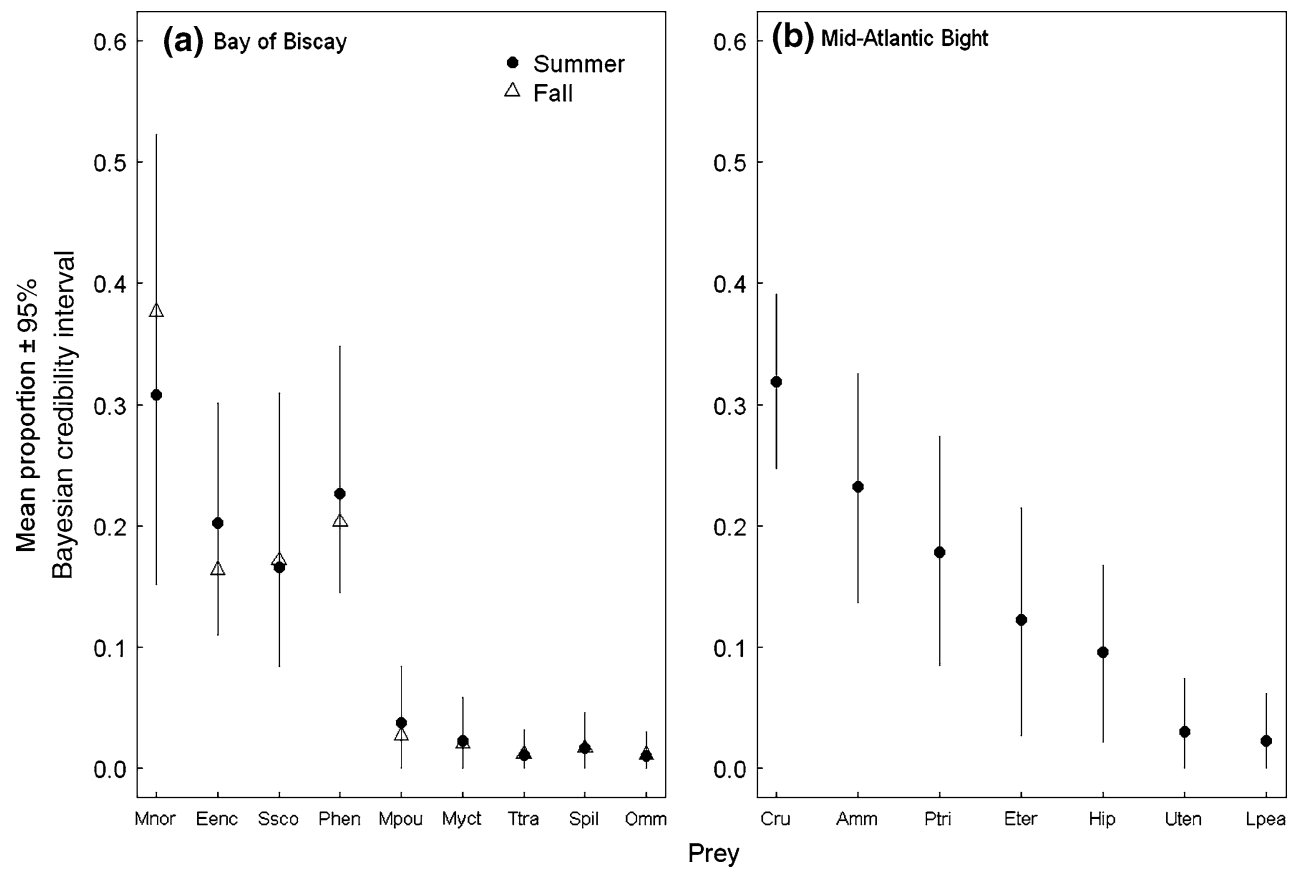

Fig. 4 Stable isotope mixing model dietary proportion estimates for Atlantic bluefin tuna (Thunnus thynnus; ABFT) from (a) the Bay of Biscay (BYB) and (b) Mid-Atlantic Bight (MAB). For the Bay of Biscay, estimates were generated separately using ABFT liver isotope data from summer and fall. For the Mid-Atlantic Bight, all liver data were pooled for a single analysis. Proportion estimates are mean $\pm 95 \%$ Bayesian credibility interval. (BYB: Mnor $=$ Meganyctiphanes norvegica ( $\mathrm{krill} ; n=4)$, Eenc $=$ Engraulis encrasicholus (European anchovy; $n=1$ ), Ssco $=$ Scomber scombrus (Atlantic

mackerel; $n=3$ ), Phen = Polybius henslowii (swimming crab; $n=1$ ), MP $=$ Micromesistius poutassou (blue whiting; $n=2$ ), Myct $=$ Myctophidae $(n=1)$, Ttra $=$ Trachurus trachurus (horse mackerel; $n=1)$, Spil = Sardina pilchardus $($ sardine; $n=1)$; MAB: Cru $=$ Crustacean $(n=3), A m m=$ Ammodytes spp. (sand lance; $n=5$ ), Ptri $=$ Peprilus triacanthus (Atlantic butterfish; $n=2$ ), Eter $=$ Etrumeus teres (round herring; $n=3$ ), Hip = Hippocampus erectus (sea horse; $n=1$ ), Uten = Urophycis tenuis (white hake; $n=3$ ), Lpea $=$ Loligo pealeii (longfin squid; $n=2$ )) 